\title{
Influence of two different membranes of equine origin on bone regeneration: histological and histometric study in rat calvaria .
}

\section{Abstract}

\section{Summary :}

Membrane materials have been widely used for guided bone regeneration (GBR). However, the bio-functional limitation of the collagen membranes leads this study to test a new equine cortical membrane ((Osteobio ${ }^{\circledR}$ Lamina) to another equine collagen membrane ((Osteobiol ${ }^{\circledR}$ Evolution) in a critical size defect $(5 \mathrm{~mm})$ of 15 Sprague Dawley rats calvaria filled with an equine biomaterial (Osteobiol ${ }^{\circledR}$ Gen-Os). An untreated group was used as a control group.

New bone fromation was found in all groups. The results of the histometric analysis showed a percentage of new bone formation $20.07 \% \pm 5.49 \%$ for the collagen group, followed by $19.87 \% \pm 5.17 \%$ for the cortical group, leading to the conclusion that there is no significant difference between the two test groups.

\section{Results}

Histological analysis showed new bone formation in all groups. The histometric analysis showed a percentage of new bone formation $20.07 \% \pm 5.49 \%$ for the COL group, followed by $19.87 \% \pm 5.17 \%$ for LAM group. The CTR group represents the smallest mean new bone formation of $8.15 \% \pm 1.43 \%$.

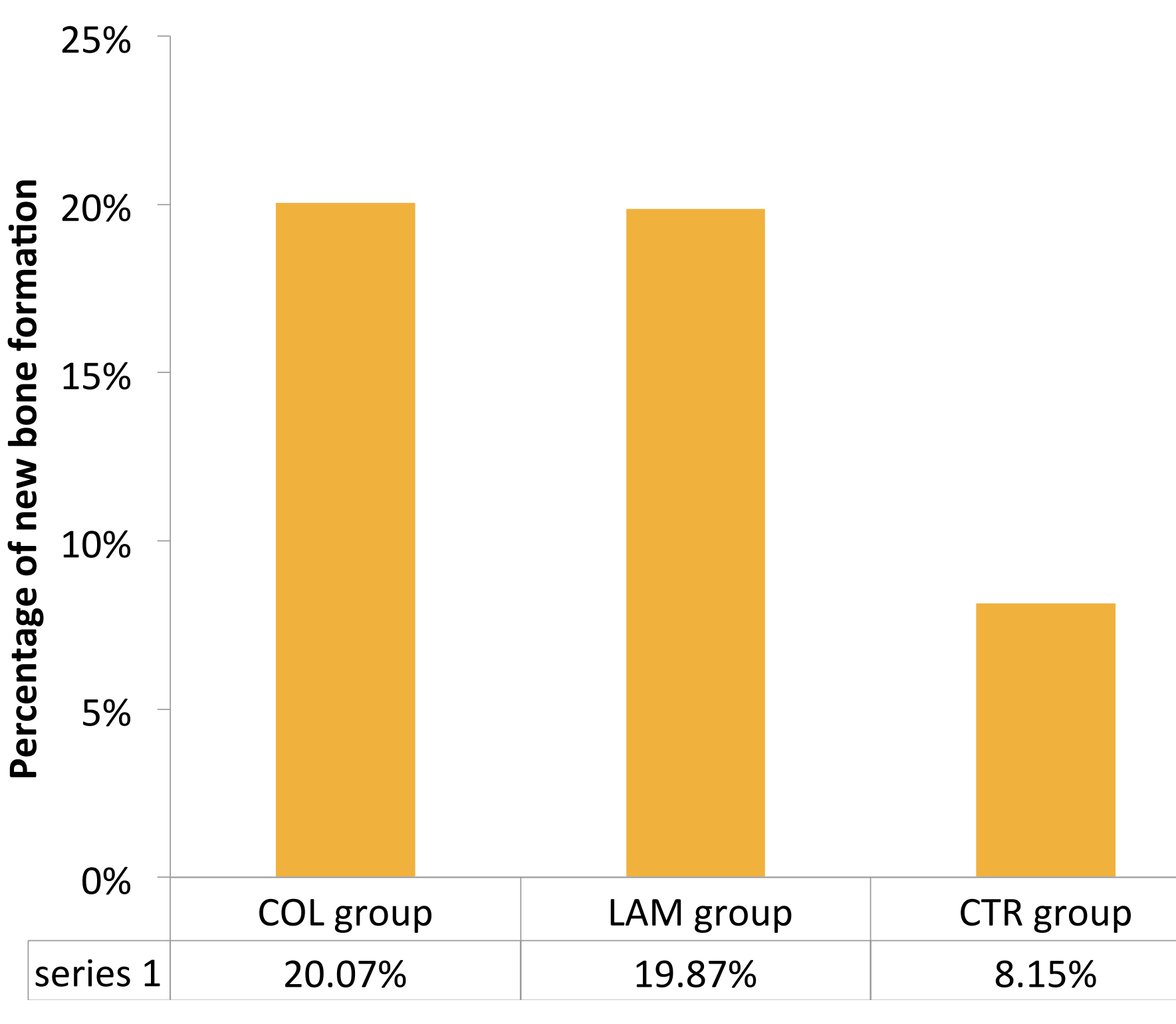

\section{Background and Aim}

Guided bone regeneration (GBR) has nowadays become an essential therapeutic procedure for bone and peri-implant defects, as well as bone augmentation procedures prior to implant placement (1). Current absorbable membrane used for guided bone regeneration (GBR) have a bio-functional limitation (2).

The aim of this study is to compare the collagen membrane of equine origin (Osteobio ${ }^{\circledR}$ Evolution) to a new equine cortical membrane (Osteobiol ${ }^{\circledR}$ Lamina) and to evaluate the new bone formation in a critical size defect in the rat calvaria filled with an equine biomaterial (Osteobiol ${ }^{\circledR}$ Gen-Os)

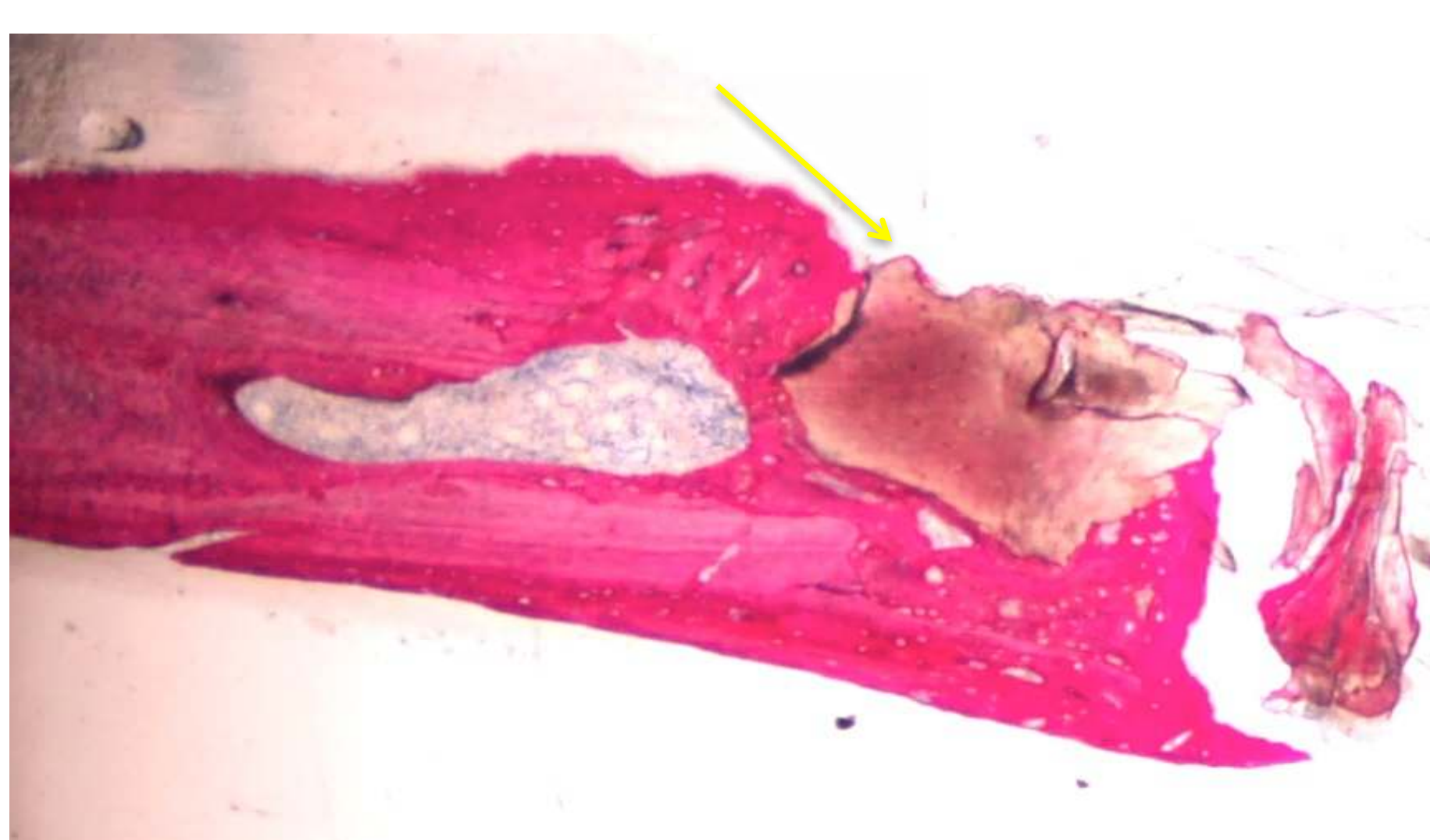

Histologic slide of LAM group showing the contact between the xenograft ( arrow) and the new immature bone.(Giemsa / Paragon X4)

\section{Conclusions}

\section{Methods and Materials}

Fifteen Sprague Dawley adults rats (250-270g) were used. Two $5 \mathrm{~mm}$ defects have been made in the calvaria (3). The rats were divided into 3 groups of 5 rats each and treated as follows. The COL group was filled with an equine xenograft covered with a resorbable collagen membrane, the LAM group was filled with an equine xenograft covered with a resorbable cortical equine membrane, in the CTR group the defects were left untreated. The animals were sacrificed at 8 weeks. The preparation of histological sections was made according to the technique of Methyl Methacrylate (MMA) for non-demineralized hard tissue.

The 2 groups test (LAM and COL) have promoted bone formation without any significant difference.

\section{References}

Becker W, Becker BE. Guided tissue regeneration for implants placedinto extraction sockets and for implant dehiscences: surgical techniques and case reports. Int J Periodontics Restorative Dent 1000, 1.376-391.

2. Wang HL, Boyapati L. "PASS" principles for predictable bone regeneration implant Dent 2006; 15:8-17

. Vajgel A, Mardas N, Farias BC, Petrie A, Cimoes R et al. A systematic review on the critical size defect model. Clin Oral Implants Res 2014 Aug; 25(8):879-93 